\title{
Simulación de Tareas Aperiódicas y Esporádicas de Tiempo Real mediante un Modelo de Eventos Discretos
}

\author{
Francisco E. Paez ${ }^{1}$, Jose M. Urriza ${ }^{1}$, Javier D. Orozco ${ }^{2}$ \\ ${ }^{1}$ Universidad Nacional de la Patagonia San Juan Bosco \\ Puerto Madryn, Argentina \\ ${ }^{2}$ Universidad Nacional del Sur \\ Bahia Blanca, Argentina \\ \{franpaez, josemurriza, jadorozco\}@gmail.com
}

\begin{abstract}
Resumen. Este trabajo presenta una extensión a un modelo de eventos discretos para el diseño de simuladores de Sistemas de Tiempo Real con tareas periódicas, incorporando tareas de tipo esporádico y aperiódico. Además, se estudia el soporte para jitter y offset. El modelo de eventos discretos elegido se ajusta a los sistemas dinámicos discretos como lo son los Sistemas de Tiempo Real El uso de simuladores para realizar comprobaciones de algoritmos, modelos, técnicas y para evaluar métricas de rendimiento, es una práctica extendida e importante en la disciplina. El modelo, es diseñado empleando la técnica de grafo de eventos.
\end{abstract}

Palabras clave: Sistemas de Tiempo Real, Simulación, Modelado, Eventos Discretos.

\section{Introducción}

La simulación por computadora es, en la actualidad, una herramienta esencial en un gran número de disciplinas. Es una herramienta importante para la obtención de nuevos resultados, acelerando el proceso de desarrollo de nuevos métodos y técnicas. Sin embargo, pocas veces se hace énfasis en la revisión y validación del software empleado para la obtención de los resultados publicados. Es evidente la importancia de validar dichos resultados, mediante el uso de soluciones software similares, por parte de otros investigadores.

Un modelo de eventos discretos para la simulación de Sistemas de Tiempo Real (STR) es presentado en [1]. Dicho modelo sirve como base sobre la cual desarrollar sistemas de simulación por computadora, y ha sido empleado con éxito en el simulador de STR del grupo de investigación de la UNPSJB ${ }^{1}$. En este trabajo se extiende dicho modelo, agregando soporte para tareas de aperiódicas y esporádicas, y exten-

1 Grupo de Investigación en STR de la Universidad Nacional de la Patagonia San Juan Bosco (UNPSJB), Sede Puerto Madryn (http://www.rtsg.unp.edu.ar). 
diendo el modelo de tareas periódicas con soporte para jitter de instanciación y offsets.

En la actualidad, un conjunto de aplicaciones y frameworks existen en la literatura para la simulación de STR. Entre los más relevantes se pueden mencionar a STRESS ([2]), PERTS ([3]), YASA ([4]), Cheddar ([5]), RealTTS ([6]) y el simulador de la Université Libre de Bruxelles ([7]). Aplicaciones como MAST ([8]) ofrecen herramientas de modelado, y otras como FORTISSIMO ([9]) presentan una plataforma para el diseño de simulaciones. Un estudio de los STR como sistemas discretos para lograr análisis temporales eficientes se encuentra en [10], en donde son modelados mediante redes de Petri. En [11] se presenta un marco general para el estudio de STR como sistemas discretos.

El presente trabajo se encuentra organizado de la siguiente manera: en la sección 2 se realiza una introducción a los STR. La sección 3 expone la simulación por eventos discretos y el modelado por grafo de eventos. Luego, en la sección 4, se realiza un resumen del modelo presentado en [1]. A continuación, las secciones 5 y 6 presentan las extensiones necesarias para el modelado de tareas aperiódicas y esporádicas. Las secciones 7,8 y 9 describen la ejecución del modelo y una implementación de referencia. Finalmente, la sección 10 expone las conclusiones y posibles trabajos futuros.

\section{$2 \quad$ Introducción a los $S T R$}

Una definición de STR ampliamente aceptada en la disciplina, es la formulada por Stankovic en [12]. La misma dice: "En los STR los resultados no solo deben ser correctos aritmética y lógicamente, si no que, además, deben producirse antes de un determinado tiempo, denominado vencimiento".

De acuerdo al vencimiento, los STR se clasifican como duros, blandos o firmes. Un STR tipo duro no tolera la perdida de ningún vencimiento bajo ninguna circunstancia. Un STR tipo blando puede permitir la perdida de algunos vencimientos. Finalmente, un STR tipo firme tipifica las pérdidas según algún criterio estadístico.

El presente trabajo utiliza un modelo de STR mono recurso, con tareas independientes y apropiables. Una tarea $i\left(\tau_{i}\right)$ periódica de tiempo real es caracterizada por su peor tiempo de ejecución $\left(C_{i}\right)$, periodo $\left(T_{i}\right)$, vencimiento $\left(D_{i}\right)$, jitter de instanciación $\left(J_{i}\right)$ y offset $\left(O f_{i}\right)$. Cada $\tau_{i}$ genera una secuencia de trabajos o instancias, siendo $j_{i, k}$, la $k$-ésima instancia. El tiempo ejecutado por una instancia $j_{i, k}$ al instante $t$, se representa como $c_{i, k}(t)$. Se considera que las tareas periódicas finalizan antes del arribo de una nueva instancia. Consecuentemente, no existen instancias previas de una misma tarea periódica esperando ser ejecutadas. Luego, un conjunto de $n$ tareas periódicas de Tiempo Real se define como $\Gamma(n)=\left\{\left(C_{1}, T_{1}, D_{1}, J_{1}, O f_{1}\right), \ldots,\left(C_{n}, T_{n}, D_{n}, J_{n}, O f_{n}\right)\right\}$.

En [13] se demostró que el peor estado de carga, para un planificador monorecurso, es aquel instante en que todas las tareas solicitan ejecución simultáneamente, y se lo denomino instante crítico. Si el planificador puede ejecutar todas las instancias de las tareas que arribaron en el instante crítico sin perder vencimientos, se considera al STR planificable. El algoritmo de planificación puede realizar una asignación está- 
tica sobre el recurso compartido, o basada en prioridades (Rate Monotonic, $R M$ [13] o Deadline Monotonic, DM [14]).

En lo que sigue, se denominarán a las tareas que cuentan con tiempos de arribo arbitrarios, como tareas aperiódicas, siendo del tipo blando. Por otro lado, a las tareas con arribos arbitrarios y con requerimientos duros se las denominarán tareas esporádicas. Para cumplir con el requerimiento temporal duro, estas últimas cuentan con un intervalo mínimo entre arribos [15].

En la literatura existen varias técnicas y métodos para la atención de estos tipos de tareas, por ejemplo buscando minimizar su tiempo de respuesta, garantizando al mismo tiempo el cumplimiento temporal de las tareas periódicas de tipo duro.

En [16] se estudió la caracterización de los STR como sistemas dinámicos, nolineales, discretos y determinísticos al ser diagramados por medio de disciplinas de prioridades fijas, tales como $R M$ o $D M$.

\section{Simulación por Eventos Discretos}

En esta sección se presenta una breve introducción a la simulación por eventos discretos, y a la técnica de modelado grafo de eventos.

La simulación por eventos discretos se aplica en el estudio de sistemas que pueden ser representados con modelos lógico-matemáticos discretos. En estos, las variables son modificadas en un determinado número finito de instantes, dentro de un intervalo determinado de tiempo ([17]). El conjunto atómico de modificaciones sobre las variables de estado del sistema, en un instante determinado, se denominado evento $(v)$.

En la simulación se emplea un reloj, $t$, que registra el tiempo actual, y una lista de eventos futuros $\Lambda$. La lista $\Lambda$, es un conjunto de tuplas $\left(t_{i}, v_{i}\right)$, siendo $t_{i} \geq t$ el instante en donde el evento $v_{i}$ será ejecutado. La misma es implementada como una cola de prioridades ordenada según los valores $t_{i}$. La simulación consiste en remover la primer tupla $\left(t_{i}, v_{i}\right)$ en $\Lambda$, actualizar $t$ con el valor $t_{i}$, y luego ejecutar las acciones asociadas al evento $v_{i}$. Una vez terminadas las acciones, se continúa con la siguiente tupla. Los procesos concurrentes pueden ser simulados mediante múltiples eventos planificados en un mismo $t_{i}$. La simulación cuenta con un conjunto de eventos iniciales, generalmente programados para $t=0$. La simulación finaliza cuando $t$ supera un valor determinado $\left(t_{\text {end }}\right)$, se encuentra un evento de finalización $\left(v_{\text {end }}\right)$ o $\Lambda$ no contiene más elementos $(\Lambda=\varnothing)$.

\subsection{Grafo de Eventos}

La técnica de grafo de eventos $([18,19])$ permite formalizar un modelo de eventos discretos. Los eventos identificados en el modelo son representados mediantes nodos, y las relaciones lógica-temporales entre estos son indicadas mediante arcos. Notar que el grafo de eventos no representa un autómata. Un grafo de eventos $\boldsymbol{M}$ consiste de:

- $\boldsymbol{S}$, el conjunto de variables y atributos que conforman el estado del sistema.

- $\boldsymbol{V}$, un conjunto de nodos que representan los eventos identificados. 
- $\boldsymbol{E}$, un conjunto de $\operatorname{arcos}$ dirigidos $e_{o d}=\left(v_{o}, v_{d}\right)$, que representan la relación lógica-temporal, entre un evento origen $\left(v_{o}\right)$ y otro destino $\left(v_{d}\right)$.

- $\boldsymbol{F}=\left\{f_{v}: \boldsymbol{S} \rightarrow \boldsymbol{S} \forall v \in \boldsymbol{V}\right\}$, funciones asociadas a cada nodo $v \in \boldsymbol{V}$, que describen los cambios en $\boldsymbol{S}$, producto de la ejecución de un evento.

- $\boldsymbol{C}=\left\{c_{o d}: \boldsymbol{S} \rightarrow\{0,1\} \forall e_{o d} \in \boldsymbol{E}\right\}$, condiciones asociadas a cada arco $e_{o d}$.

- $\boldsymbol{D}=\left\{\delta_{\text {od }} \in \mathbb{R}_{0}^{+} \forall e_{\text {od }} \in \boldsymbol{E}\right\}$ deltas asociados a cada arco $e_{o d}$.

- $\boldsymbol{A}=\left\{\boldsymbol{A}_{e}, e_{o d} \in E\right\}$, atributos asociados al arco $e_{o d}$ y $\boldsymbol{B}=\left\{\boldsymbol{B}_{v}, v \in V\right\}$, parámetros asociados al evento $v$.

El modelo es especificado como $\boldsymbol{M}=(\boldsymbol{V}, \boldsymbol{E}, \boldsymbol{S}, \boldsymbol{F}, \boldsymbol{C}, \boldsymbol{D}, \boldsymbol{A}, \boldsymbol{B})$. Cada arco $e_{o d}$ se recorre si la condición $c_{o d}$ es válida luego de ejecutar el evento $v_{o}$. Esto equivale a programar el evento $v_{d}$ en el instante $t+\delta_{o d}\left(\delta_{o d}\right.$ es el delta asociado a $\left.e_{o d}\right)$. El conjunto de atributos $\boldsymbol{A}_{\boldsymbol{e}}$ del arco $e_{o d}$, son los argumentos que el evento $v_{d}$ espera recibir como parámetros $\left(\boldsymbol{B}_{v}\right)$, y son opcionales. Tanto $\Lambda$ como $t$ están asociados con la ejecución de la simulación de $\boldsymbol{M}$, y no son partes del modelo. Esta técnica es empleada en [1], para realizar un modelo de $S T R$, que es resumido a continuación.

\section{Modelo de eventos discretos para un STR de tareas periódicas}

A continuación se resume el modelo de eventos discretos desarrollado en [1]. Este modelo identifica seis eventos asociados a tareas periódicas. Estos son:

- Inicio ( $v_{0}$, prioridad 0): Corresponde al inicio de la simulación.

- Terminación $\left(v_{\text {end }}\right.$, prioridad 1): Indica la finalización de la simulación.

- Arribo ( $v_{1}$, prioridad 4): Arribo de una nueva instancia de una tarea periódica.

- Ejecución ( $v_{2}$, prioridad 5): Ejecución de la instancia con mayor prioridad.

- Finalización ( $v_{3}$, prioridad 2): Finaliza la ejecución de una instancia.

- Desalojo $\left(v_{4}\right.$, prioridad 3): Posible desalojo de una instancia del recurso.

El evento Arribo recibe como parámetro la nueva instancia $j_{i, k}$ de una $\tau_{i}$. Luego, habrá tantos eventos $v_{1}$ como tareas tenga el STR. Las prioridades asignadas permiten ordenar correctamente la ejecución de eventos planificados para un mismo instante $t_{i}$. La máxima prioridad es cero. Las relaciones entre estos eventos se especifican mediante los siguientes arcos:

- $e_{01}$ : planifica el arribo de las primeras instancias de las tareas periódicas.

- $e_{11}$ : programa el arribo de la próxima instancia de una tarea periódica (evento $v_{1}$ ).

- $e_{12}$ : planifica la ejecución de la instancia de mayor prioridad (evento $v_{2}$ ).

- $e_{23}$ : programa la finalización de la instancia en ejecución (evento $v_{3}$ ).

- $e_{24}$ : planifica el posible desalojo de la instancia en ejecución (evento $v_{4}$ ).

- $e_{32}$ : programa la ejecución de la instancia de mayor prioridad (evento $v_{2}$ ).

Cada arco tiene asociada una condición, que de cumplirse indica que se debe programar el evento en el otro extremo del mismo:

- $c_{11}$ : La instancia previa de la tarea $\tau_{i}$ no excedió su tiempo de ejecución. 
- $c_{12}$ : No existen otros eventos de tipo $v_{1}$ en $\Lambda$ para el instante actual.

- $c_{23}$ : La instancia finaliza su ejecución antes del arribo del próximo evento $v_{1}$.

- $c_{24}$ : La instancia actual no finaliza antes del arribo del próximo evento $v_{1}$.

- $c_{32}$ : No existen eventos de tipo $v_{1}$ en $\Lambda$ para el instante actual, y existe al menos una instancia sin finalizar en la cola de listos del planificador.

De existir más de un evento $v_{1}$ en un mismo instante, la condición $c_{32}$ evita la duplicación de un evento $v_{2}$. Las condiciones $c_{23}$ y $c_{24}$ son mutuamente excluyentes.

Si una condición se evalúa positivamente, el evento destino del arco al que dicha condición corresponde, se programa para un instante $t_{i} \geq t$. El modelo de grafo de eventos especifica dicho instante mediante un delta $\delta_{o d} \geq 0$, uno por arco, tal que $t_{i}=t+\delta_{o d}$. Luego, los deltas del modelo son:

- $\delta_{11}=\left\lfloor t+T_{i} / T_{i}\right\rfloor T_{i}-t$, siendo $\left\lfloor t+T_{i} / T_{i}\right\rfloor T_{i}$ el instante donde ocurre el próximo arribo de una instancia de la tarea $\tau_{i}$.

- $\delta_{23}=C_{i}-c_{i}(t)$, que es el tiempo remanente de ejecución de la instancia de mayor prioridad en la cola de listos del planificador.

- $\delta_{24}=t_{1}-t$, siendo $t_{1}$ el instante donde está programado el evento $v_{1}$ más próximo.

- $\delta_{01}=\delta_{12}=\delta_{32}=0$, ya que el evento destino se planifica para el instante actual.

El arco $e_{24}$ se recorre cuando un evento Arribo $\left(v_{1}\right)$ interrumpe la ejecución de la instancia actual. Por lo tanto el evento Desalojo $\left(v_{4}\right)$ es planificado en el tiempo de arribo de dicho evento, $t_{1}$, y se tiene que $\delta_{24}=t_{1}-t$.

\section{$5 \quad$ Modelado de tareas aperiódicas y esporádicas}

Existen dos opciones para agregar las tareas aperiódicas y esporádicas al modelo. Una primera opción es modificar la lógica del evento Arribo para que administre estos tipos de tareas, en conjunto con las tareas periódicas. Esto evita incrementar el número de eventos en el modelo. Como contrapartida, la lógica del evento Arribo se torna más compleja. Una segunda opción es agregar nuevos eventos para el arribo de tareas aperiódicas y esporádicas. Esto incrementa la complejidad del modelo, pero permite un mayor detalle y control. En este trabajo se empleará esta última alternativa. Los siguientes eventos son agregados:

- ArriboA ( $v_{6}$, prioridad 4): Arribo de una nueva instancia de una tarea aperiódica.

- ArriboE (v $v_{7}$, prioridad 4): Arribo de una nueva instancia de una tarea esporádica.

Ambos eventos, tienen como parámetro la instancia de $\tau_{i}$ que debe arribar. El evento Arribo $\left(v_{1}\right)$ destinado a las tareas periódicas, se renombra como ArriboP. Se denominará a los eventos $v_{1}, v_{6} \mathrm{y} v_{7}$ como eventos de tipo Arribo. Los nuevos arcos son:

- $e_{06} \mathrm{y} e_{07}$, que programan el primer evento de $v_{6} \mathrm{y} v_{7}$ en la simulación.

- $e_{66}$ y $e_{77}$, que planifican el próximo arribo del evento $v_{6}$ o $v_{7}$, respectivamente.

- $e_{62}$ y $e_{72}$, planifica la ejecución de la instancia con mayor prioridad (evento $v_{2}$ ). 
Las condiciones asociadas a los nuevos arcos son:

- $c_{77}$ : La instancia previa de la tarea esporádica no excedió su tiempo de ejecución.

- $c_{72}$ y $c_{62}$, ambas verifican si no existen otros eventos de tipo Arribo en $\Lambda$ para el instante actual.

Los deltas asociados con los nuevos arcos son:

- $\delta_{66}$, es el próximo tiempo de arribo de la instancia de la tarea aperiódica.

- $\delta_{77}$, es el próximo tiempo de arribo de la instancia de la tarea esporádica, debe respectar el tiempo mínimo entre arribos de la tarea.

- $\delta_{72}=\delta_{62}=0$, ya que el evento $v_{2}$ se planifica para el instante actual.

Los eventos $v_{6}$ y $v_{7}$ agregan la instancia de la tarea aperiódica o esporádica que recibieron como parámetro al planificador. Como la ejecución de estas tareas (por medio de servidores, uso de slack, o relegación a ejecución en background) es decisión del planificador (que es activado por un evento $v_{2}$ ), y no del modelo de eventos, el orden en que son procesados los eventos de tipo Arribo $\left(v_{1}, v_{6}\right.$ o $\left.v_{7}\right)$ programados en un mismo instante, no es determinante. Luego, todos cuentan con la misma prioridad (4). Como las instancias de una tarea aperiódica pueden acumularse no es necesario una condición asociada al arco $e_{66}$ que controle la programación de nuevos arribos.

\section{Jitter y Offset}

El Offset de una tarea $\tau_{i}\left(O f_{i}\right)$ es el máximo desplazamiento que sufre desde su tiempo de arribo, respecto del instante crítico ([20,21]). Es útil, por ejemplo, para modelar relaciones de precedencia, evitando que una tarea sea instanciada antes que otra haya terminado su ejecución. Debido a que es una perturbación que afecta a todas las instancias de una tarea, se puede modelar sumando el valor del offset al delta utilizado para la planificación del evento ArriboP. Luego el delta será $\delta_{11}+O f_{\mathrm{i}}$.

El jitter de instanciación $\left(J_{i}\right)$, en su caso más común, es el tiempo insumido por el Sistema Operativo de Tiempo Real en tener lista para ejecución la tarea $\tau_{i}$ (desde su arribo). Este tiempo es acotado y no afecta al período de la tarea $\left(T_{i}\right)$. La presencia de jitter en una tarea puede generar una mayor interferencia en las tareas de menor prioridad, lo que puede resultar en pérdidas de vencimientos de las mismas. Estos casos son estudiados en [22, 23, 24, 25], entre otros.

Para modelar el efecto del jitter sobre las instancias, se tendrá en cuenta el hecho que, hasta que el jitter no haya expirado, la instancia no será visible para el planificador. De esta manera, antes de programar el evento ArriboP, para el arribo de la próxima instancia de $\tau_{i}$, se calcula el valor de jitter de dicha instancia. Luego, ArriboP será programado en el instante $t+\delta_{11}+J_{i}$. Luego, para agregar el soporte para jitter y offset, se debe modificar el delta asociado al arco $e_{11}$, siendo su nuevo valor $\delta_{11}=\left\lfloor t+T_{i} / T_{i}\right\rfloor T_{i}+J_{i}+O f_{i}-t$. 
A continuación se presenta el grafo para el nuevo modelo de eventos discretos. A fin de simplificar el diagrama, se omite el evento Terminación, así como los deltas con un valor igual a cero. Los $\operatorname{arcos} e_{11}, e_{66} \mathrm{y} e_{77}$ también se omiten por claridad.

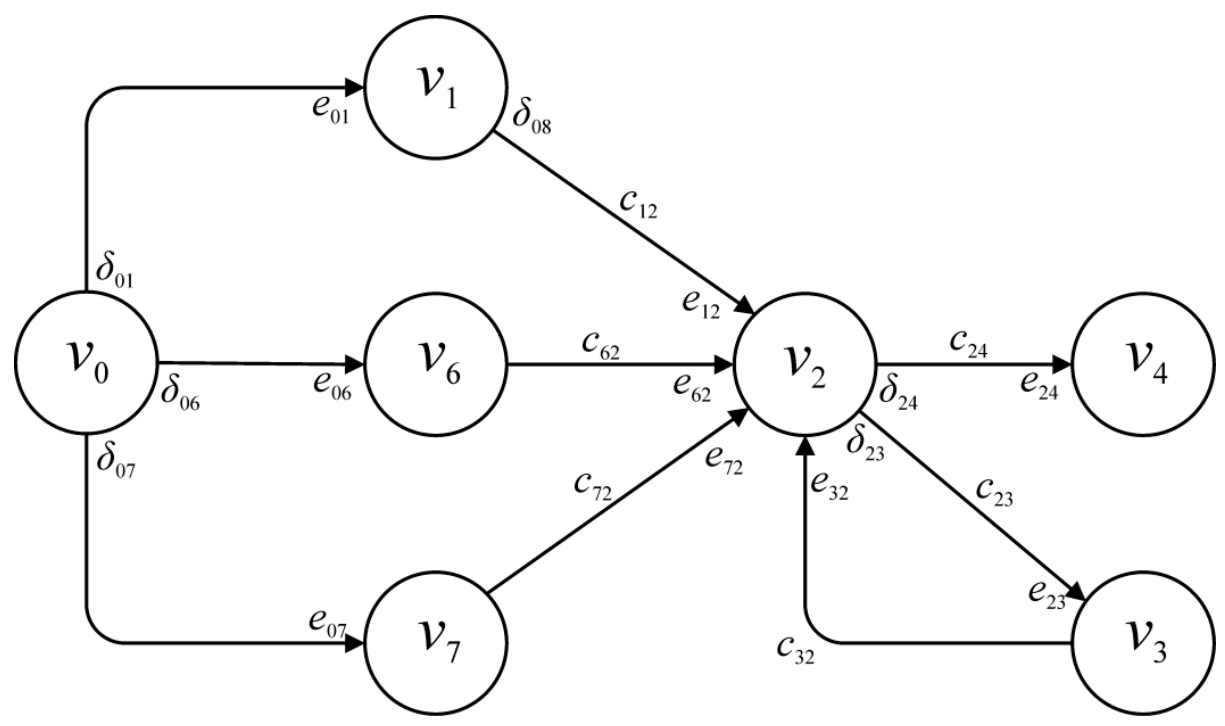

Figura 1. Grafo de Eventos parcial para el STR.

\section{$7 \quad$ Análisis de la Cola de Eventos Futuros}

La cola de eventos futuros $(\Lambda)$ contiene los próximos eventos a ejecutarse. De acuerdo a los arcos y condiciones entre los eventos $v_{2}, v_{3}$ y $v_{4}$, se puede observar que para cualquier instante, existe en $\Lambda$ una única instancia de $v_{2}, v_{3}$ o $v_{4}$.

Consideremos un evento ArriboP $\left(v_{1}\right)$. La ejecución de un evento de este tipo, programa en $\Lambda$ su próxima instancia. De esta manera, y recordando que se remueve el evento de la lista antes de su ejecución, existe en $\Lambda$ a lo sumo una instancia de $v_{1}$. Como cada evento ArriboP representa una tarea en particular del STR simulado, para un $S T R$ con $n$ tareas, habrá como máximo $n$ eventos $v_{1}$ en $\Lambda$ en cualquier instante.

La situación para los eventos de tipo ArriboE $\left(v_{7}\right)$ es análoga. Luego, dado un STR con $n$ tareas periódicas y $m$ tareas esporádicas, habrá a lo sumo $n+m$ eventos $v_{2}$ y $v_{7}$, en $\Lambda$.

La situación para el evento Arribo $A$ es similar. El próximo evento $v_{6}$ puede ser generado por la ejecución del evento anterior. $\mathrm{O}$ bien todos los eventos ArriboA esperados pueden ser programados al inicio de la simulación.

Notar también que, durante la ejecución de un evento $v_{2}, v_{3}$ o $v_{4}$, el próximo evento en $\Lambda$ es siempre de tipo Arribo $\left(v_{1}, v_{6}\right.$ o $\left.v_{7}\right)$. Luego, se pueden simplificar las condiciones $c_{12}, c_{23}, c_{24} \mathrm{y} c_{32}$, sustituyendo $v_{1}$ por el tiempo de ocurrencia del siguiente evento en $\Lambda$. 


\section{$8 \quad$ Ejecución del modelo}

A continuación se describe genéricamente la ejecución del modelo, y las acciones a realizar por parte de los eventos.

El evento Inicialización $\left(v_{0}\right)$ crea, por cada tarea del STR, un evento del tipo Arribo $\left(v_{1}, v_{6}\right.$ o $\left.v_{7}\right)$ apropiado. Estos son los eventos iniciales de la simulación. Se inicializa el reloj, generalmente en $t=0$. Otras actividades de setup se pueden realizar en $v_{0}$ (por ejemplo, cálculo de slack en el instante crítico, peores tiempos de respuesta, etc.). Luego, la simulación toma el primer evento en $\Lambda$, y lo ejecuta.

La ejecución de un evento de tipo Arribo $\left(v_{1}, v_{6}\right.$ o $\left.v_{7}\right)$ agrega una nueva instancia de una tarea de tiempo real al planificador, y programa su próxima ejecución en $\Lambda$, en un instante futuro, de acuerdo al delta correspondiente. Luego, de cumplirse alguna de las condiciones $c_{12}, c_{62}, \mathrm{o} c_{72}$ (según el tipo de evento Arribo ejecutado) un evento Ejecución $\left(v_{2}\right)$ es planificado en el instante actual.

El evento Ejecución $\left(v_{2}\right)$, invoca la rutina del planificador a fin de simular la ejecución de la instancia con mayor prioridad en la cola de listos. Luego, si se satisface la condición $c_{23}$, un evento Finalización $\left(v_{3}\right)$ es planificado en $t+\delta_{23}$. Caso contrario la condición $c_{24}$ será válida, y un evento Desalojo $\left(v_{4}\right)$ se programa para $t+\delta_{24}$.

El evento Finalización $\left(v_{3}\right)$ invoca las rutinas del planificador que simulan el fin de ejecución de una instancia. A continuación, de ser válida la condición $c_{32}$, un nuevo evento $v_{2}$ se programa en el instante actual. De esta manera se continúa ejecutando las tareas de la cola de listos del planificador. De manera similar, el evento Desalojo $\left(v_{4}\right)$ invoca las rutinas requeridas para realizar el desalojo de una tarea. Como el evento Desalojo se programa en el mismo instante que un evento de tipo Arribo, este último se encargará de generar un evento $v_{2}$.

El evento Terminación $\left(v_{5}\right)$, programado en $t_{\text {end }}$, libera los recursos utilizados, e invoca las rutinas auxiliares necesarias, por ejemplo de generación de reportes.

Se hace notar, que el planificador se ejecuta únicamente a pedido de los distintos eventos. Por lo tanto, su única responsabilidad es mantener la cola de tareas listas, y decidir que tarea debe ejecutar según la política de planificabilidad simulada.

\section{Implementación}

A continuación se ofrecerá una síntesis de una implementación mediante la librería SSJ ([26]) para Java. Esta librería ofrece dos clases principales para la simulación por eventos discretos, Simulator y Event.

La clase Simulator representa el ejecutivo de simulación. Administra el reloj, la lista de eventos $\Lambda$, y ofrece métodos para iniciar, finalizar y reiniciar la simulación. Cada evento del modelo se implementa como una clase que extiende Event (Init, ArrivalP, ArrivalE, ArrivalA, Run, End y Preempt), y sobrecarga el método actions() con las acciones a realizar al ejecutar el evento. La clase Event es una clase abstracta que representa a un evento genérico.

Se presume que existe una estructura de datos con el STR a simular, y clases auxiliares que implementan el planificador (Scheduler) y los métodos o técnicas a evaluar. 
El método actions() de la clase Init $\left(v_{0}\right)$, crea las instancias iniciales de ArrivalP $\left(v_{1}\right)$, ArrivalA $\left(v_{6}\right)$ y ArrivalE $\left(v_{7}\right)$. Estas instancias son programadas mediante el método schedule(delay), de la clase Event. Las clases Arrival, desde el método actions(), agregan la nueva instancia de una tarea $\tau_{i}$ al planificador. Cada clase comprueba su condición $c_{\text {od }}$ asociada, y de cumplirse, planifica una nueva instancia de Run $\left(v_{2}\right)$ en el instante actual.

La clase Run invocará, por ejemplo, Scheduler.runTask(), que simulará la ejecución de la instancia de mayor prioridad. Luego, según se cumplan las condiciones $c_{23}$ o $c_{24}$, se programará una instancia End $\left(v_{3}\right)$ o Preempt $\left(v_{4}\right)$, que invocarán los métodos necesarios en el planificador, según corresponda. Por ejemplo, End invocará Scheduler.finishTask(), y Preempt el método Scheduler.preemptTask(). Así la lógica del planificador se desacopla de la de los eventos. La clase End, de cumplirse la condición $c_{32}$, planifica una nueva instancia de la clase $R u n$, para el instante actual. La clase Simulator ofrece métodos para inspeccionar $\Lambda$, a fin de comprobar las diferentes condiciones $c_{o d}$.

\section{Conclusiones y Trabajos Futuros}

Se ha presentado una extensión al modelo de eventos discretos presentado en [1], agregando soporte para la simulación de tareas aperiódicas y esporádicas, así como también para los casos de jitter y offset de las tarea periódicas de tiempo real.

El modelo resultante brinda una base formal para el desarrollo de un simulador de $S T R$, pudiendo hacerse uso de cualquiera de los paquetes DES disponibles en la actualidad. A su vez, el presente trabajo sirve de base para futuras extensiones del mismo, como por ejemplo para la simulación de STR heterogéneos.

\section{Referencias}

[1] F. E. Paez, J. M. Urriza, C. E. Buckle, S. Lucas, and J. D. Orozco, "A Discrete Event Model for Real Time System Simulation," Journal of Computer Science and Technology (JCS\&T), vol. 12, pp. 99-103, 2012.

[2] N. C. Audsley, A. Burns, M. F. Richardson, and A. J. Wellings, "STRESS: a simulator for hard real-time systems," Softw. Pract. Exper., vol. 24, pp. 543-564, 1994.

[3] J. W. S. Liu, J. L. Redondo, Z. Deng, T. S. Tia, R. Bettati, A. Silberman, et al., "PERTS: A prototyping environment for real-time systems," in Real-Time Systems Symposium, 1993., Proceedings., 1993, pp. 184-188.

[4] F. Golatowski, J. Hildebrandt, J. Blumenthal, and D. Timmermann, "Framework for validation, test and analysis of real-time scheduling algorithms and scheduler implementations," in Rapid System Prototyping, 2002. Proceedings. 13th IEEE International Workshop on, 2002, pp. 146-152.

[5] F. Singhoff, J. Legrand, L. Nana, and L. Marcé, "Cheddar: a flexible real time scheduling framework," Ada Lett., vol. XXIV, pp. 1-8, 2004. 
[6] A. Diaz, R. Batista, and O. Castro, "Realtss: a real-time scheduling simulator," in Electrical and Electronics Engineering, 2007. ICEEE 2007. 4th International Conference on, 2007, pp. 165-168.

[7] S. d. Vroey, J. Goossens, and C. Hernalsteen, "A Generic Simulator of Real-Time Scheduling Algorithms," presented at the Proceedings of the 29th Annual Simulation Symposium (SS '96), 1996.

[8] M. Gonzalez Harbour, J. J. Gutierrez Garcia, J. C. Palencia Gutierrez, and J. M. Drake Moyano, "MAST: Modeling and analysis suite for real time applications," in Real-Time Systems, 13th Euromicro Conference on, 2001., 2001, pp. 125-134.

[9] T. Kramp, M. Adrian, and R. Koster, "An Open Framework for Real-Time Scheduling Simulation," in Proceedings of the 15 IPDPS 2000 Workshops on Parallel and Distributed Processing, 2000, pp. 766-772.

[10] J. Teich, L. Thiele, and E. A. Lee, "Modeling and simulation of heterogeneous real-time systems based on a deterministic discrete event model," in System Synthesis, 1995., Proceedings of the Eighth International Symposium on, 1995, pp. 156-161.

[11] E. A. Lee, "Modeling concurrent real-time processes using discrete events," Ann. Softw. Eng., vol. 7, pp. 25-45, 1999.

[12] J. A. Stankovic, "Misconceptions About Real-Time Computing: A Serius Problem for Next-Generations Systems," IEEE Computer, vol. Octubre, pp. 10-19, 1988.

[13] C. L. Liu and J. W. Layland, "Scheduling Algorithms for Multiprogramming in a Hard Real-Time Environment," Journal of the ACM, vol. 20, pp. 46-61, 1973.

[14] N. C. Audsley, A. Burns, M. F. Richarson, and A. J. Wellings, "Hard Real-Time Scheduling: The Deadline Monotonic Approach," in Proceedings 8th IEEE Workshop on Real-Time Operating Systems and Software, Atlanta, GA, USA 1991.

[15] B. Sprunt, "Aperiodic Task Scheduling for Real-Time Systems," Doctor in Computer Engineering, Department of Electrical and Computer Engineering, Carnegie Mellon University, Carnegie Mellon University, 1990.

[16] J. M. Urriza, R. Cayssials, and J. D. Orozco, "Modelado de Sistemas de Tiempo Real Planificados por RM o DM: Caracterización y Análisis," in XXXIV Conferencia Latinoamericana de Informática, CLEI 2008, Santa Fe, Argentina, 2008, pp. 1435-1444.

[17] A. M. Law and W. D. Keaton, Simulation Modelling and Analysis, 2nd ed.: McGraw-Hill Higher Education, 1997.

[18] L. Schruben, "Simulation modeling with event graphs," Commun. ACM, vol. 26, pp. 957963, 1983.

[19] E. L. Savage, L. W. Schruben, and E. Yücesan, "On the Generality of Event-Graph Models," INFORMS J. on Computing, vol. 17, pp. 3-9, 2005.

[20] J. C. Palencia and M. Gonzalez Harbour, "Schedulability analysis for tasks with static and dynamic offsets," in Real-Time Systems Symposium, 1998. Proceedings., The 19th IEEE, 1998, pp. 26-37.

[21] I. Bate and A. Burns, "Schedulability analysis of fixed priority real-time systems with offsets," in Real-Time Systems, 1997. Proceedings., Ninth Euromicro Workshop on, 1997, pp. 153-160.

[22] K. W. Tindell, "Fixed Priority Scheduling of Hard Real-Time Systems," Doctor of Philosophy, Department of Computer Science, University of York, 1993. 
2do Simposio Argentino de Informatica Industrial, SII 2013

[23] N. C. Audsley, A. Burns, M. F. Richardson, K. Tindell, and A. J. Wellings, "Applying New Scheduling Theory to Static Priority Preemptive Scheduling," Software Engineering Journal, vol. 8, pp. 284-292, 1993.

[24] P. Richard and J. Goossens, "Approximating Response Times of Static-Priority Tasks with Release Jitters," in Euromicro Conference on Real-Time Systems. WIP Dresden, Germany, 2006, p. 4.

[25] O. Redell and M. Torngren, "Calculating exact worst case response times for static priority scheduled tasks with offsets and jitter," in Real-Time and Embedded Technology and Applications Symposium, 2002. Proceedings. Eighth IEEE, 2002, pp. 164-172.

[26] P. L'Ecuyer and E. Buist, "Simulation in Java with SSJ," in Simulation Conference, 2005 Proceedings of the Winter, 2005, p. 10 pp. 